\title{
Computational Studies to Establish the Broad range Potentiality of Violacein- The Anti-Cancerous Drug
}

\author{
Dr. P. Mary Anupama, Swathi Silarapu, Mantriah \\ (Department of chemical Engineering, Anil Neerukonda Institute of technology and Sciences, Andhra Pradesh, \\ India.531162, drmaryanupama@gmail.com)
}

\begin{abstract}
Docking studies are proved to be an effective tool that facilitates the structural diversity of products to be connected in an organized manner. The aim of the present investigation is to prove that violacein is a broad range anti-cancerous drug as compared to other available drugs. In this study protein structure similarity of different proteins that are responsible for causing various types of cancer were studied using BLAST. Among all the proteins under study, maximum structural similarity of $75 \%$ identity was seen between the protein receptors PU1 and $p 53$ and $71 \%$ similarity between RB and FGFR. Their efficiency of their binding properties of violacein, the anti-cancerous drug was determined using docking studies with ArgusLab and SwissDock. Violacein has exhibited good binding properties with almost all cancer causing protein. Maximum best ligand pose energy value of $-9.61 \mathrm{Kcal} / \mathrm{mol}$ was seen with Mutant FLT3 cancer protein receptor among all the mutant proteins under investigation. Hence it is concluded that Violacein is a broad range drug that can be used for treatment of various cancers.
\end{abstract}

Keywords - Anti-Cancerous drug, ArgusLab, Cancer, Docking, SwissDock and Violacein

\section{Introduction}

Most Cancers are outlines as the uncontrolled growth of abnormal cells as they cannot be subjected to the check points that can identify and decide whether the cells to divide and differentiate or to die. Thus they build up a level of self-governance from the signs and create uncontrolled development forming tumors.

Cancer can be recognized to defective protein-protein interactions (PPIs), therefore this type of intermolecular event is a highly attractive target in drug discovery. The belief that targeting PPIs is an unsuitable strategy in drug design has been challenged by recent successful cases, such as the development AMG-232, a MDM2-p53 inhibitor, currently in Phase II clinical trials for cancer therapy [1]. Drugs are one of the effective treatments for cancer and they are classified according to their site of action and specific point in the biosynthetic pathways of bio-molecules like cytotoxic antibodies, anti-metabolites, anti-tumor antibodies, plant alkaloids, biological agents and DNA linking agents.

There are numerous drugs available for cancer remedy and most of them inhibit the DNA synthesis or a few other syntheses in the cell growth cycle. There are number of cancerous drugs available for the treatment of cancer like Cycloplasmide a drug used to treat cancers and autoimmune ailment. It is a powerful drug in treating the disease but has been eradicated due to its toxicity. It works by using the T-regulatory cells (CD4+ CD25+ T cells) in naive and tumor benign hosts. And induction of T-cellular boom factors consisting of type 1 IFNS [2], Cisplastin an intravenous drug that works with the aid of interfering with DNA replication that kills the proliferating cells. After the administration the two chloride ligands are slowly displaced with the aid of the water to shape aqua complicated [3], Methotrexate a chemotherapy agent and immune device suppressant works by using inhibiting the dihydrofolate reductase that is involved in tetrahydrofolate synthesis this is crucial for purines and pyrimidines synthesis hence inhibiting the synthesis of DNA, RNA and proteins [4], Vincristine used to treat many cancers like acute lymphocytic leukemia, acute myeloid leukemia works by binding to the tubulin protein stopping the cell from separating its chromosomes during the metaphase then undergoes apoptosis. But an overuse of Vincristine leads to over expression of the P-glycoprotein pump [5].

Various pigments like Varamine, Violacein, Amphimedine, Fascaplysin, Monascin, Chinikomycin, and so forth were discovered with anticancer activity and the anticancer activity is docked using Glide [6]. Violacein is one such pigment isolated from Chromobacterium violaceum and has diverse biological properties because of its anti leukemic activity that has increased interest owing to its important biological activities and pharmacological potential as an anti-cancerous agent.

It has effectiveness on four types of colon cancer cell lines that cause apoptosis in HC60 leukemic cells that was followed by activation of caspase 8 transcription of nuclear factor Kappa B and p38 MAP kinase activation. Violacein showed a efficiency in number of cell lines in both neoplastic and hematological malignant origins and it is most effective against MOLT-4 leukemia that is found to inhibit the growth and proliferation of colorectal cancer cell lines. The activity is also enhanced by the hypoxia-induced cells [7] and the increase of 
mitochondrial membrane potential in MRC-5 and HeLa cells also suggests that mitochondrial membrane hyper polarization might be the main cause of cell death triggered by violacein [8].

Thus the current study is focused on determining the activity of potent dye violacein for its anticancerous activity by using molecular docking techniques.

\subsection{Drug Discovery}

Drug discovery is a process of finding a new medicine for a therapeutic use. One of the most capable methods to find out the new drug is to find out the target protein interactions with randomly chosen compounds that are the part of compound libraries. Thus, the accurate prediction of the binding modes between the ligand and protein is of fundamental importance in modern structure-based drug design. Computer-based molecular modeling aims to speed up drug discoveries by predicting potential effectiveness of ligand-protein interactions. Molecular docking is one such method of a structural based drug design.

\subsection{Molecular Docking}

Molecular docking is a tool that facilitates the structural molecular biology and computer assisted drum design. The aim of this is to identify the major binding modes of the ligand to that of a three dimensional structure. Thus this finds out the intermolecular complex formed between the two molecules [9]. Molecular docking uses the scoring function that can predict the binding strength, complex energy and can also evaluate the binding affinity between the protein and the ligand [10] that may result in the activation or inhibition of the enzyme. because of its ability to predict, degree of accuracy and the conformation of small molecule ligands with the target binding site [11] it is used as a most common method used in drug design. Furthermore molecular docking algorithms also execute rankings of the docked compounds based on the affinity of the ligand-receptor complex [12]. Detailed understanding of the principles that direct the nature of different interactions provide an outline for designing the drug of the therapeutic target. For this a variety of docking methods has been employed. These methods provide a ranking of the ligand and their ability to interact with the target. The optimal binding is measured by the scoring function. During computational docking a pose is generated, scored and compared to the previous pose. This decides the previous pose to be considered or deleted based on the score. This scoring iterates till it achieves to an endpoint and identifies the perfect fit.

Numerous software packages have been developed with the implementation of various molecular docking algorithms based on different search methods [13]. The present work of molecular docking has been done using commercially available software's ArgusLab and SwissDock.

ArgusLab is a molecular modeling, graphics, and drug designing program based on genetic algorithm. It is implemented with exhaustive search methods, the Argus Dock docking engine and AScore scoring function [14]. It is also capable of performing molecular geometry calculations and molecular structure visualization.

SwissDock is a docking web server that can detect the structure of the target protein, as well as that of the ligand automatically prepared for docking and additionally the cumbersome syntax of the docking engine hides a clean web interface that provides reasonable alternative sets of parameters along with sample input files [15].

\subsection{Computational Methodology}

\section{Materials and Methods}

\subsubsection{Data Set}

Three-dimensional (3D) experimentally known protein-ligand complexes were obtained from Brookhaven Protein Data Bank (PDB). Biological databases like PDB(protein data bank), pubchem, drug bank, KEGG (Kyto Encyclopedia of Genes and Genomes) can be used for the same.

\subsubsection{Generation of Violacein 3D structure:}

ChemSpider server was used for generating 3D structure of violacein (Fig 1) and SMILES notation of violacein was found in chemspider that was used to translate this 3D structure by SMILES translator online server, employing JAVA based structure applet in chemSpider. Then the geometric was reframed using the Universal Force Field (UFF), molecular mechanics and final geometry was performed by Semi empirical Quantum mechanics method (QM). Finally, violacein structure was saved as MOL file for further docking procedure [16]. 


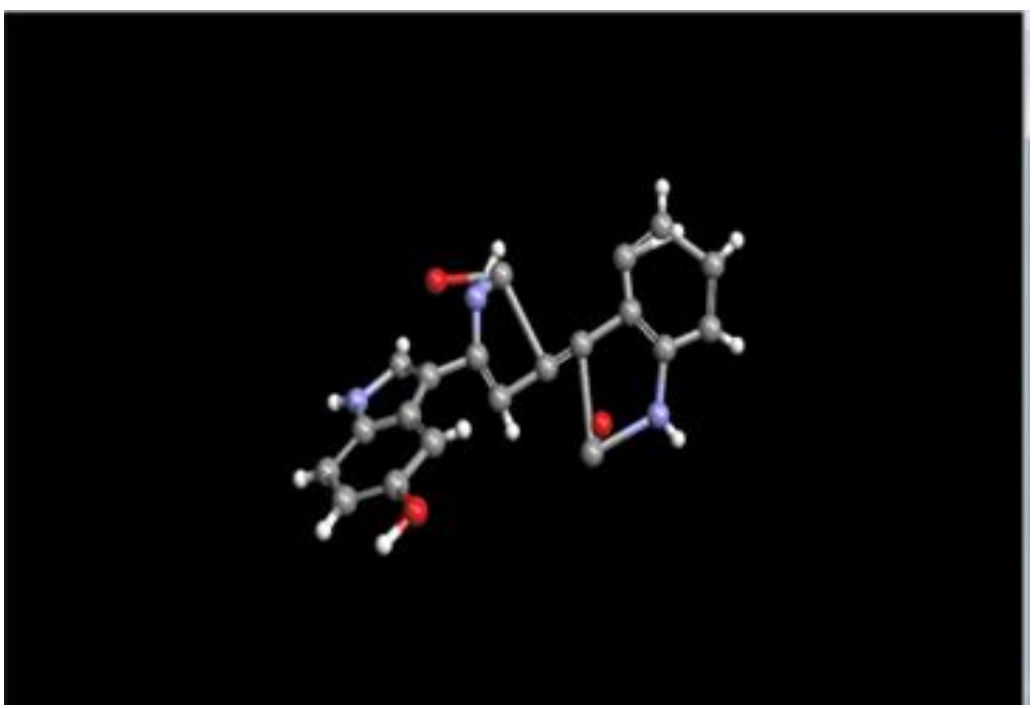

Fig 1.3D structure of violacein using ChemSpider

\subsubsection{Collection of cancer effecting proteins and prediction of protein structure similarity:}

The cancer effecting proteins were retrieved from the KEGG (Kyoto Encyclopedia of Genes and Genomes) a predominant database for retrieving the information about Cancer path ways. Ten categories of cancer pathways were retrieved from KEGG and most effective were listed. (Table 1). Structural similarity of these proteins corresponding to the ten types of cancers was predicted using BLAST and FASTA format.

Table 1. Proteins involved in causing various types of cancers

\begin{tabular}{|l|l|}
\hline Cancer & Protein \\
\hline Pancreatic cancer & $\begin{array}{l}\text { KRas(Kirsten rat sarcoma viral oncogene homolog), } \mathrm{P}^{53} \text { (Tumor Protein 53), } \\
\mathrm{P}^{16} \text { (cyclin-dependent kinase inhibitor). }\end{array}$ \\
\hline Colorectal cancer & $\begin{array}{l}\text { Beta-catenine, KRas, APC(adenomatosis polyposis coli 2), DCC(deleted in } \\
\text { colorectal carcinoma), TGF } \beta \text { RII(transforming growth factor, beta receptor II), } \\
\mathrm{P}^{53} \text { (Tumor Protein 53). }\end{array}$ \\
\hline Glioma & PTEN(phosphatase and tensin homolog), MDM2( ubiquitin protein ligase). \\
\hline Thyroid cancer & TRK(TRK-fused gene), Beta-catenine. \\
\hline Acute myeloid leukemia. & $\begin{array}{l}\text { FLT 3(fms-related tyrosine kinase 3), PU1(spleen focus forming virus (SFFV) } \\
\text { proviral integration oncogene). }\end{array}$ \\
\hline Melanoma & $\begin{array}{l}\text { MITF(microphthalmia-associated transcription factor), PTEN(phosphatase and } \\
\text { tensin homolog), } \mathrm{P}^{53} \text { (Tumor Protein 53). }\end{array}$ \\
\hline Bladder Cancer & $\begin{array}{l}\text { Hras(Harvey rat sarcoma viral oncogene homolog), fgfr(fibroblast growth } \\
\text { factor receptor 3 ), RB(retino blastoma 1), }\end{array}$ \\
\hline Prostate cancer & AR(androgen receptor), PTEN(phosphatase and tensin homolog), \\
\hline Endometrial cancer & $\begin{array}{l}\text { KRas(Kirsten rat sarcoma viral oncogene homolog), Beta catenine, } \\
\text { PTEN(phosphatase and tensin homolog), } \mathrm{P}^{53}\end{array}$ \\
\hline Small cell lung cancer & RB(retinoblastoma1),PTEN(phosphatase and tensin homolog). \\
\hline
\end{tabular}

\subsection{Docking methodology}

\subsubsection{ArgusLab}

Argus Lab is docking software that was implemented with shape-based search algorithm using "Argus Dock" exhaustive search docking function of ArgusLab consisting of grid resolution of $20 \AA$. The ligand and binding site groups were created using 910GOL. Then the 3D structure of violacein is opened in the ArgusLab that is then introduced and docking calculation was allowed to run using shape-based search algorithm and scoring function (Fig 2). Docking was performed for cancer causing proteins in all ten categories of cancers with violacein. 


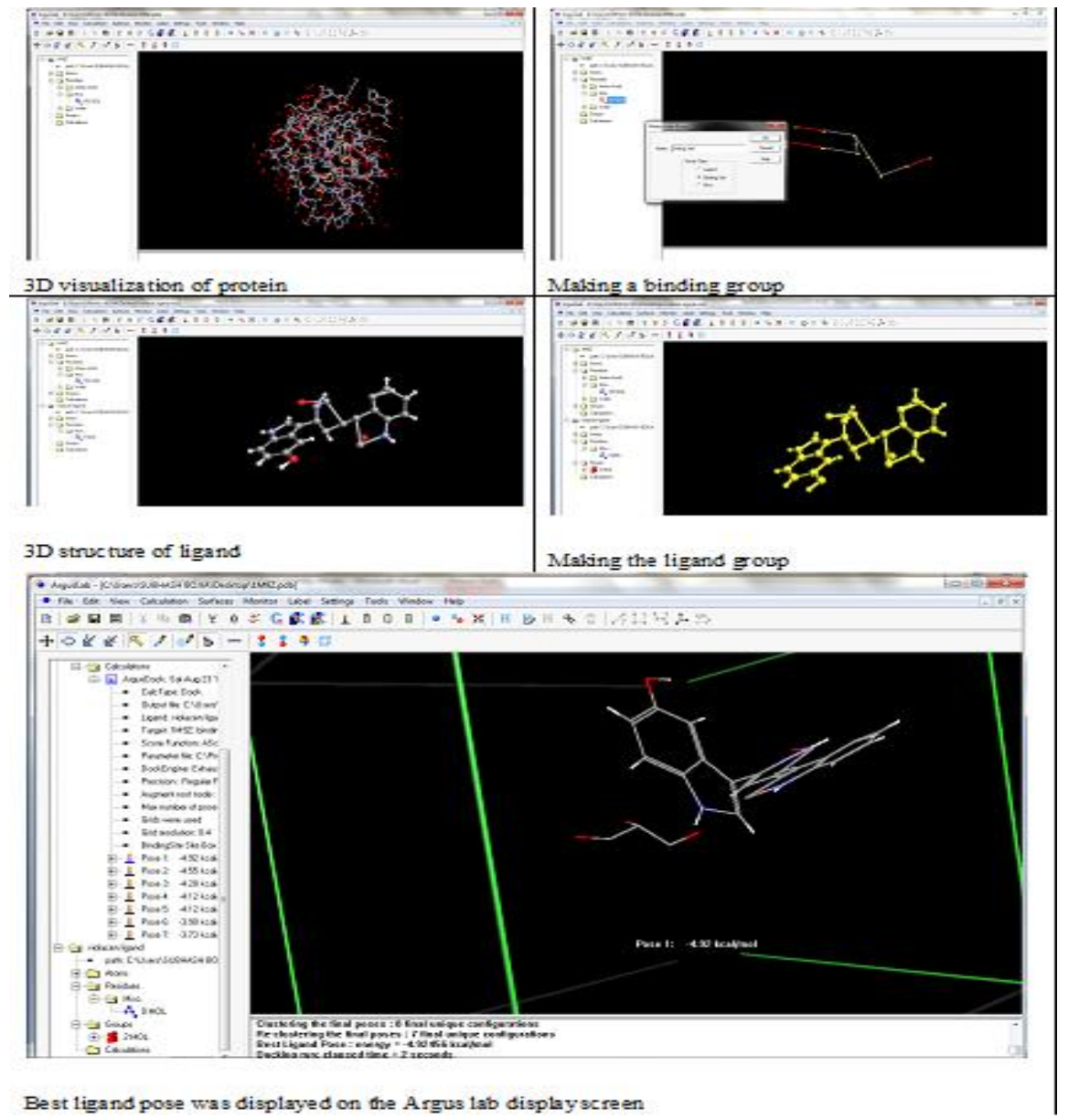

Fig 2: ligand pose of violacein obtained using ArgusLab

\subsubsection{SwissDock}

Desirable protein and ligand were selected to perform docking studies in SwissDock. The resultant was shown in the (Fig 3). Cancer protein receptors of Ten types of cancers were docked with Violacein ligand. The ligand pose energy values of all the poses of protein was retervied and the best ligand pose energy value was selected

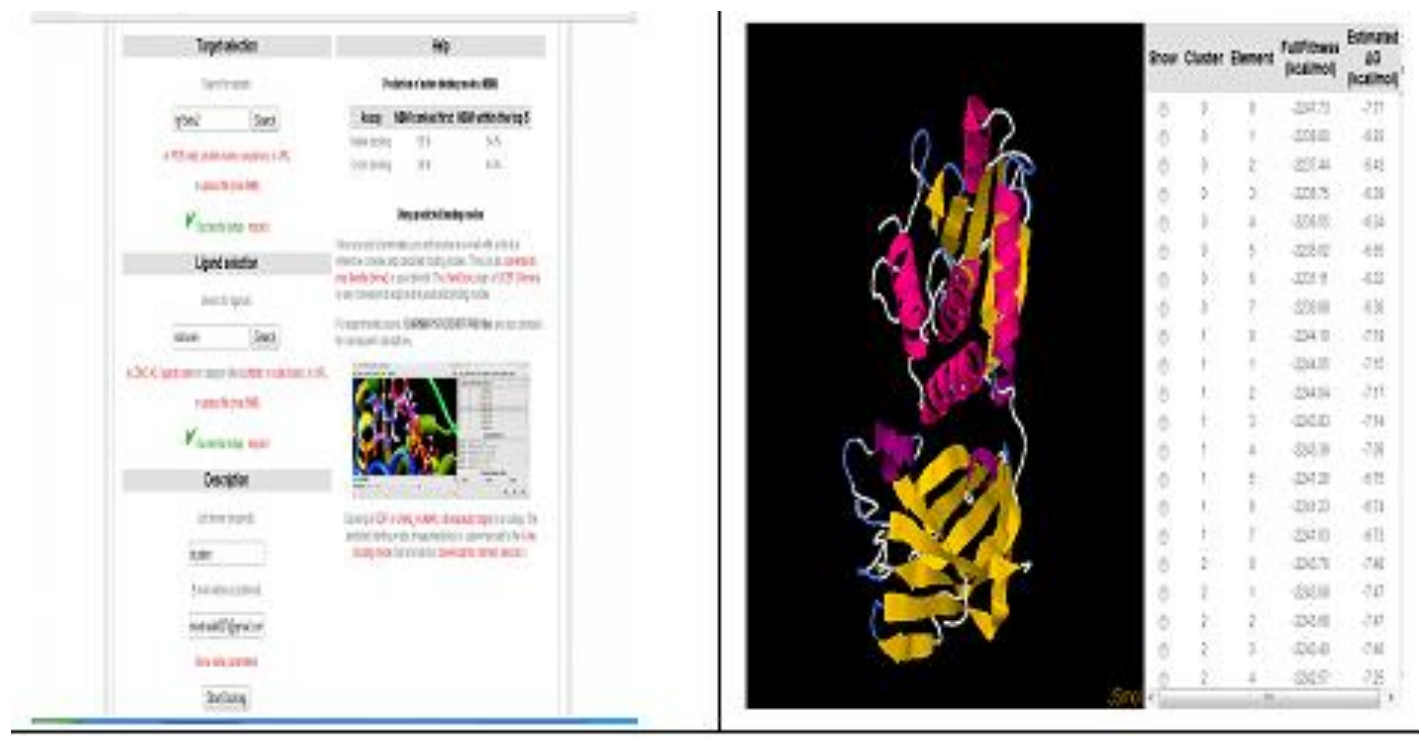

Fig. 3 ligand pose obtained using SwissDoc 


\subsection{Structure similarity studies}

\section{Results and Discussion}

BLAST was used to identify the structural similarity of the proteins that were causing cancer. This enables to dock the proteins with the best suitable drug. Seventeen types of proteins that are responsible for ten types of cancers were evaluated for their structural similarity the results of which are shown in Fig 4.

\begin{tabular}{|c|c|c|c|c|c|c|c|c|c|c|c|c|c|c|c|c|c|}
\hline K-RAS & 100 & & & & & & & & & & & & & & & & \\
\hline P. 53 & No & 100 & & & & & & & & & & & & & & & \\
\hline P 16 & 63 & 23 & 100 & & & & & & & & & & & & & & \\
\hline$\beta$ Cat & 23 & NO & 60 & 100 & & & & & & & & & & & & & \\
\hline APC & NO & 38 & 57 & 26 & 100 & & & & & & & & & & & & \\
\hline$D C C$ & NO & 29 & 25 & 50 & 43 & 100 & & & & & & & & & & & \\
\hline TGF & NO & 50 & NO & NO. & NO. & 67 & 100 & & & & & & & & & & \\
\hline PTEN & 28 & 38 & 33 & No. & 43 & 38 & 29 & 100 & & & & & & & & & \\
\hline MDM-2 & NO & NO & NO & No. & 32 & 57 & 60 & 31 & 100 & & & & & & & & \\
\hline TRK & NO & 27 & 28 & 32 & 31 & 46 & NO & 25 & NO & 100 & & & & & & & \\
\hline FLT3 & 34 & 40 & 47 & No. & 42 & 38 & NO & 57 & NO & 29 & 100 & & & & & & \\
\hline PU1 & 27 & 75 & 67 & 23 & 28 & NO & NO & 33 & NO & NO & 18 & 100 & & & & & \\
\hline MITF & 36 & No & No & No & 35 & NO & 50 & NO & NO & NO & NO & NO & 100 & & & & \\
\hline HRAS & 36 & 38 & NO & 23 & NO & 24 & NO & 40 & 45 & 38 & NO & 55 & NO & 100 & & & \\
\hline FGFR & NO & 23 & 27 & 50 & NO & 24 & NO & 44 & 33 & 31 & 29 & NO & NO & NO & 100 & & \\
\hline RB & 50 & 33 & 30 & 55 & 28 & 33 & 22 & 22 & 50 & 27 & 26 & 56 & 28 & 28 & 71 & 100 & \\
\hline \multirow[t]{2}{*}{ AR } & NO & 17 & 20 & 32 & 50 & NO & NO & 29 & NO & 42 & 35 & 50 & NO & 25 & 36 & 26 & 100 \\
\hline & K-RAS & P. 53 & P16 & $\beta$ Cat & APC & $D C C$ & TGF & PTEN & MDM-2 & TRK & FLT3 & PU1 & MITF & HRAS & FGFR & RB & AR \\
\hline
\end{tabular}

Note: No indicates no acceptable ligand pose.

Fig 4 Protein structural similarity of different cancer causing proteins

Among all the proteins under study, maximum structural similarity was seen between the protein receptors PU1 and p53 of $75 \%$ identity and between RB and FGFR, with $71 \%$ similarity.

\subsection{Docking Studies:}

In the docking study with Argus lab it is observed that violacein had exhibited best binding pose with FLT3 of $(-9.16 \mathrm{Kcal} / \mathrm{mol})$ that causes acute myeloid leukemia followed by Beta-catenine $(-8.57 \mathrm{Kcal} / \mathrm{mol})$ and $\mathrm{k}-\mathrm{Ras}$ of $(-8.18 \mathrm{Kcal} / \mathrm{mol})$. Among all the cancer causing proteins under stud with violacein, it has exhibited good binding property to most of the proteins except for those causing prostate cancer.

\subsubsection{SwissDock results:}

From the ArgusLab it is clearly seen that Violacein has exhibited good binding properties. So it is considered for the SwissDock study. The results were tabulated in table along with the comparison with that of the ArgusLab results in (Table 2). 
Table 2: Binding energies of various Proteins with Violacein involved in causing various types of cancers using ArgusLab and SwissDoc.

\begin{tabular}{|c|c|c|c|}
\hline Name of the Protein & PDB file code & $\begin{array}{l}\text { Violacein } \\
\text { (Aurgus lab } \\
\text { (K.Cal/mol) } \\
\end{array}$ & $\begin{array}{l}\text { Violacein } \\
\text { (Swiss Docking) } \\
\text { (K.cal/mol) }\end{array}$ \\
\hline \multicolumn{4}{|l|}{ 1.Pancreatic Cancer. } \\
\hline KRas (Kirsten rat sarcoma viral oncogene homolog) & 4LRW & -8.18 & -7.57 \\
\hline $\mathrm{P}^{53}$ (Tumor Protein 53) & 1TUP & -4.08 & -7.82 \\
\hline $\mathrm{P}^{16}$ (cyclin-dependent kinase inhibitor) & 2R3R & -6.76 & -7.95 \\
\hline \multicolumn{4}{|l|}{ 2.Colorectal Cancer } \\
\hline Beta-catenine & 3SLA & -8.57 & $\mathrm{NO}$ \\
\hline KRas & & -8.18 & -7.57 \\
\hline APC(adenomatosis polyposis coli 2) & $3 \mathrm{NMX}$ & -6.34 & -7.58 \\
\hline DCC(deleted in colorectal carcinoma) & 2EP7 & -5.99 & NO \\
\hline $\begin{array}{l}\text { TGF } \beta \text { RII(transforming growth factor, beta receptor } \\
\text { II) }\end{array}$ & $1 \mathrm{M} 9 \mathrm{Z}$ & -6.25 & -7.62 \\
\hline P 53 & 1TUP & -4.08 & -7.57 \\
\hline \multicolumn{4}{|l|}{ 3.Glioma } \\
\hline PTEN(phosphatase and tensin homolog) & 1D5R & -7.16 & -7.76 \\
\hline MDM2( ubiquitin protein ligase) & $2 \mathrm{MDN}$ & -4.85 & -7.49 \\
\hline \multicolumn{4}{|l|}{ 4.Thyroid Cancer } \\
\hline TRK(TRK-fused gene) & 3V5Q & -6.66 & $\mathrm{NO}$ \\
\hline Beta-catenine & 3SLA & -8.57 & $\mathrm{NO}$ \\
\hline \multicolumn{4}{|l|}{ 5.Acute Myeloid leukemia } \\
\hline FLT 3(fms-related tyrosine kinase 3) & 1XAC & -9.61 & -7.23 \\
\hline $\begin{array}{l}\text { PU1(spleen focus forming virus (SFFV) proviral } \\
\text { integration oncogene) }\end{array}$ & 1PUE & -5.41 & $\mathrm{NO}$ \\
\hline \multicolumn{4}{|l|}{ 6.Melanoma } \\
\hline MITF(microphthalmia-associated transcription factor) & 4ATH & -7.41 & -5.93 \\
\hline PTEN(phosphatase and tensin homolog) & 1D5R & -7.16 & -7.76 \\
\hline $\mathrm{P}^{53}$ (Tumor Protein 53) & 1TUP & -4.08 & -7.82 \\
\hline \multicolumn{4}{|l|}{ 7.Bladder Cancer } \\
\hline Hras(Harvey rat sarcoma viral oncogene homolog) & 2VHS & -6.96 & -8.02 \\
\hline FGFR(fibroblast growth factor receptor 3 ) & 3GRW & -6.46 & -7.81 \\
\hline RB(retinoblastoma 1) & 4ELJ & -6.76 & -7.89 \\
\hline \multicolumn{4}{|l|}{ 8.Prostate Cancer } \\
\hline $\mathrm{AR}$ (androgen receptor) & 1GS4 & $\mathrm{NO}$ & -8.01 \\
\hline PTEN(phosphatase and tensin homolog) & 1D5R & -7.16 & -7.76 \\
\hline \multicolumn{4}{|l|}{ 9.Endometrial Cancer. } \\
\hline KRas & 3GFT & -8.18 & 7.57 \\
\hline Beta-catenine & $1 \mathrm{JDH}$ & -8.51 & $\mathrm{NO}$ \\
\hline PTEN(phosphatase and tensin homolog) & 1D5R & -7.16 & -7.76 \\
\hline $\mathrm{P}^{53}$ (Tumor Protein 53) & 1TUP & -4.08 & -7.82 \\
\hline \multicolumn{4}{|l|}{ 10.Small cell lung cancer. } \\
\hline RB(retinoblastoma 1) & 4ELJ & -6.76 & -7.89 \\
\hline PTEN(phosphatase and tensin homolog) & 1D5R & -7.16 & -7.76 \\
\hline
\end{tabular}

Though the results between the ArgusLab and SwissDock vary in terms of the binding affinity, Violacein has exhibited maximum binding property with 11 proteins that are responsible for 9 cancers

\section{Conclusion}

The proteins under study though have exhibited very less structural similarity, and yet all of them have shown some affinity for violacein. Docking studies with the ArgusLab and SwissDock revealed that it has affinity with many proteins that are responsible for causing cancers like colorectal cancer, pancreatic cancer, thyroid cancer and endometrial cancer indicate its potential application as a broad range anti-cancerous drug.

\section{Acknowledgements}

We express our sincere thanks to the management of ANITS for providing the infrastructure and facilities and the Head of the Department Prof. S.Subba Rao for his consistent support.

\section{References}

[1] Sun. D, Li .Z, Rew .Y, Gribble. M, Bartberger. MD., Beck .HP, Canon .J, Chen .A, Chen. X, Chow. D, Deignan. J, Duquette. J, Eksterowicz, J, Fisher.B, Fox. B, Fu. J, Gonzalez. AZ, Gonzalez-Lopez De Turiso F, Houze. JB, Huang. X, Jiang. M, Jin. L, Kayser, F, Liu. J.J, Lo,MC, Long. AM, Lucas. B, McGee. LR., McIntosh. J, Mihalic. J, Oliner. JD, Osgood. T, Peterson. ML, Roveto. P, Saiki. AY, Shaffer. P, Toteva. M, Wang. Y, Wang, YC., Wortman, S., Yakowec, P., Yan, X., Ye, Q., Yu, D.Yu, M., Zhao, X., Zhou, J., Zhu, J., Olson, SH., Medina, JC. Discovery of AMG 232, a potent, selective, and orally bioavailable MDM2-p53 inhibitor in clinical development. J. Med. Chem. 57, 2014, 1454-1472. 
[2] Sistigu. A, Viaud. S, Chaput. N. Immunomodulatory effects of cyclophosphamide and implementations for vaccine design. Semin Immunopathol, 33, 2011, 369-383.

[3] Timothy C Johnstone, Kogularamanan Suntharalingam, Stephen J Lippard. The Next Generation of Platinum Drugs: Targeted Pt(II) Agents, Nanoparticle Delivery, and Pt(IV) Prodrugs. Chem. Rev. 116, 2016, 3436-3486.

[4] Rajagopalan. P, T. Ravi., Zhang Zhiquan, Mc. Court, Lynn, Dwyer Mary, Benkovic Stephen J, Hammes G G. Interaction of dihydrofolate reductase with methotrexate: Ensemble and single-molecule kinetics. Proceedings of the National Academy of Sciences. 99, 2002, 13481-13486.

[5] Sears. J, Boger. D. Total Synthesis of Vinblastine, Related Natural Products, and Key Analogues and Development of Inspired Methodology Suitable for the Systematic Study of Their Structure-Function Properties. Accounts of Chemical Research. 48, 2015, $653-662$.

[6] Vikas Sharma, prabodh chander Sharma, Vipin kumar. In silico Molecular docking analysis of Natural Pyridoacridines as Anticancer Agents. Advances in Chemistry (2016)

[7] Hashimi. SM, Xu. T, Wei, MQ. Violacein anticancer activity is enhanced under hypoxia. Oncol Rep. 33, $2015,1731-1736$.

[8] Angélica Maria de Sousa Leal, Jana Dara Freires de Queiroz, Silvia Regina Batistuzzo de Medeiros, Tatjana Keesen de Souza Lima, Lucymara Fassarella Agnez-Lim. Violacein induces cell death by triggering mitochondrial membrane hyper polarization in vitro. BMC Microbiology. 15 (115),2015.

[9] Lengauer. T, Rarey. M. Computational methods for biomolecular docking. Current opinion in Structural Biology. 6, 1996, 402406.

[10] Kitchen. DB, Decomez. H, Furr. JR, Bajorath, J. Docking and scoring in virtual screening for drug discovery: methods and applications. Nature reviews. Drug discovery. 3, 2004, 935-949.

[11] Meng. X.Y, Zhang. H.X, Mezei. M, Cui. M. Molecular docking: A powerful approach for structure-based drug discovery. Curr. Comput. Aided Drug Des. 7, 2011, 146-157.

[12] Huang. S.Y, Zou. X. Advances and challenges in protein-ligand docking. Int. J. Mol.sci. 11, 2010, 3016-3034.

[13] M. A. Thompson, Molecular docking using ArgusLab, an efficient shape-based search algorithm and AScore scoring function, Proceedings of the ACS Meeting, Philadelphia, Pa, USA, 2004, 172.

[14] R. Wang, X. Fang, Y. Lu, S. Wang. The PDB bind database: collection of binding affinities for protein-ligand complexes with known three-dimensional structures. Journal of Medicinal Chemistry, 47 2004, 2977-2980.

[15] Grosdidier. A, Zoete. V, Michielin. O. SwissDock, a protein-small molecule docking web service based EA Dock DSS. Nucleic Acids Res. 39, 2011, 270-277.

[16] Meshram, Nilesh. Virtual Screening and Docking Exploration on Estrogen receptor. Indian Journal of Biotechnology. 11, 2012, 385-395. 